\title{
BMJ Open HealthPathways implementation in a New Zealand health region: a qualitative study using the Consolidated Framework for Implementation Research
}

Tim Stokes, ${ }^{1}$ Emma Tumilty, ${ }^{1,2}$ Fiona Doolan-Noble, ${ }^{1}$ Robin Gauld ${ }^{3}$

To cite: Stokes T, Tumilty E, Doolan-Noble F, et al. HealthPathways implementation in a New Zealand health region: a qualitative study using the Consolidated Framework for Implementation Research. BMJ Open 2018;8:e025094. doi:10.1136/ bmjopen-2018-025094

- Prepublication history and additional material for this paper are available online. To view these files, please visit the journal online (http://dx.doi org/10.1136/bmjopen-2018025094).

Received 29 June 2018 Revised 3 November 2018 Accepted 9 November 2018

\section{Check for updates}

C C Author(s) (or their employer(s)) 2018. Re-use permitted under CC BY-NC. No commercial re-use. See rights and permissions. Published by BMJ.

${ }^{1}$ Department of General Practice and Rural Health, Dunedin School of Medicine, University of Otago, Dunedin, New Zealand ${ }^{2}$ Institute for Translational Sciences, University of Texas Medical Branch, Galveston, Texas, USA

${ }^{3}$ Otago Business School, University of Otago, Dunedin, Otago, New Zealand

Correspondence to Professor Tim Stokes; tim.stokes@otago.ac.nz

\section{ABSTRACT}

Objectives To explore the process of implementation of an online health information web-based portal and referral system (HealthPathways) using implementation science theory: the Consolidated Framework for Implementation Research (CFIR).

Setting Southern Health Region of New Zealand (Otago and Southland).

Participants Key Informants (providers and planners of healthcare) $(n=10)$ who were either involved in the process of implementing HealthPathways or who were intended end-users of HealthPathways.

Methods Semistructured interviews were undertaken. A deductive thematic analysis using CFIR was conducted using the framework method.

Results CFIR postulates that for an intervention to be implemented successfully, account must be taken of the intervention's core components and the adaptable periphery. The core component of HealthPathways - the web portal and referral system that contains a large number of localised clinical care pathways-had been addressed well by the product developers. Little attention had, however, been paid to addressing the adaptable periphery (adaptable elements, structures and systems related to HealthPathways and the organisation into which it was being implemented); it was seen as sufficient just to deliver the web portal and referral system and the set of clinical care pathways as developed to effect successful implementation. In terms of CFIR's 'inner setting' corporate and professional cultures, the implementation climate and readiness for implementation were not properly addressed during implementation. There were also multiple failures of the implementation process (eg, lack of planning and engagement with clinicians). As a consequence, implementation of HealthPathways was highly problematic.

Conclusions The use of CFIR has furthered our understanding of the factors needed for the successful implementation of a complex health intervention (HealthPathways) in the New Zealand health system. Those charged with implementing complex health interventions should always consider the local context within which they will be implemented and tailor their implementation strategy to address these.

\section{Strengths and limitations of this study}

- This is the first process evaluation of implementing HealthPathways in New Zealand using qualitative methods.

- The use of implementation science theory (Consolidated Framework for Implementation Research) allowed us to 'unpack' the reasons why the implementation of HealthPathways in the Southern Region of New Zealand was so challenging.

- The study focused on a single health region as it was designed in partnership with the local health system to provide context-specific findings that would be of benefit to the local health system.

- We were only able to recruit small number of participants who were not involved with the implementation process.

\section{INTRODUCTION}

The New Zealand (NZ) health system, in common with those of other jurisdictions, struggles to provide integration of health services across primary and secondary care. ${ }^{1}$

One important quality improvement initiative promoted internationally since the $1980 \mathrm{~s}$ to improve healthcare integration is care pathways. Care pathways have five key characteristics; they: (1) provide a structured multidisciplinary plan of care; (2) translate national evidence-based clinical guidelines into local structures; (3) detail the components of care required in an algorithm or pathway; (4) provide a time frame or criterion-based progression through the health system; and (5) aim to standardise care for a specific clinical condition in a specific local population. ${ }^{2}$ In NZ, a key adaptation has been the embedding of web-based clinical care pathways into general practice hospital electronic referral management systems (ERMS). The use of care pathways in NZ has been championed by Canterbury District Health Board (DHB) who, in 2008, developed an online health 
information web-based portal (containing the individual care pathways for specific clinical conditions) and referral system called HealthPathways. ${ }^{3}$ Currently, HealthPathways provides health practitioners with guidance and a referral pathway for over 550 clinical conditions (https:// www.healthpathwayscommunity.org/About.aspx). There has been rapid adoption of HealthPathways and similar systems across NZ's DHBs and in Australia. ${ }^{4-7}$ Its advocates claim that HealthPathways is an important means of achieving healthcare integration, ${ }^{89}$ which has been a NZ government priority since the 2009 publication of Better Sooner More Convenient. ${ }^{10}$ This claim is supported by an emerging evidence base that suggests that HealthPathway use is associated with an improvement in referral quality from primary care to secondary care and more timely access to secondary care. ${ }^{3}$ End users of HealthPathways also report, in online surveys ${ }^{9}$ and qualitative case studies, ${ }^{5}{ }^{11}$ that HealthPathways improved their knowledge of local services and changed their clinical management decisions.

In the Southern Health Region of NZ's South Island (Otago and Southland), the Southern DHB and the WellSouth Primary Health Network (Primary Health Organisation $(\mathrm{PHO})$ ) are working to further health service integration through Alliance South, ${ }^{12}$ which is a contractual alliance between the two organisations aimed at improving care coordination and integration. In 2013, Alliance South embarked on the implementation of the Canterbury HealthPathways in the Southern RegionSouthern HealthPathways. The approach used clinical editors-general practitioners (GPs) who consulted with selected Southern Region secondary care clinicians (hospital specialists) — to localise/adapt each individual Canterbury clinical care pathway for use in the Southern health region: by 2017, over 400 individual clinical care pathways had been localised and could be accessed through the Southern HealthPathways web portal and referral system (https://southern.healthpathways.org. $\mathrm{nz} /$ ). The focus was on the technical development of a suite of adapted pathways. In 2016, a team of researchers from the Dunedin School of Medicine, University of Otago, partnered with the local health system through Alliance South to conduct a mixed methods evaluation of the Southern HealthPathways programme. The rationale for this university-health service partnership was to build evaluation capacity in the Southern Region, and the HealthPathways initiative was identified as a strategic priority for evaluation by Alliance South.

We report here the findings of the qualitative process evaluation. One key challenge in evaluating the implementation of HealthPathways is that they can be conceptualised as a complex healthcare intervention, ${ }^{13}$ and thus without a qualitative process evaluation, it is not possible to determine which aspects of the intervention in a defined healthcare context are likely to lead to its success. Our study aim was therefore to understand the process by which Southern HealthPathways were being implemented using a commonly used implementation science theory: the Consolidated Framework for Implementation Research (CFIR). ${ }^{1415}$

\section{METHODS \\ Consolidated Framework for Implementation Research}

In understanding how quality improvement initiatives work, there is increasing emphasis on the use of theory drawn from the social sciences to better develop quality improvement interventions, optimise their design and identify aspects of context necessary for their success. ${ }^{16} 17$ The CFIR is a 'meta-theoretical' framework that provides an overarching typology of implementation. It offers a comprehensive, standardised list of constructs that allow researchers to identify variables that are most relevant to a particular intervention. ${ }^{14}$ The CFIR addresses intervention delivery (context, implementation and mechanisms of action) through 26 constructs organised into five major domains: intervention characteristics (eight constructs), outer setting (four constructs), inner setting (five constructs), characteristics of the individuals involved (five constructs) and the process of implementation (four constructs) (see box 1). CFIR has been widely used to inform qualitative process evaluations across a range of complex interventions. ${ }^{15}$

\section{Design and sampling}

Semistructured interviews were conducted by ET between May and November 2016 with key informants (providers and planners of care) from the Southern DHB catchment area (Otago and Southland). The Southern DHB is the southernmost DHB in NZ and is responsible for planning, funding and providing health and disability services to a population of over 300000 and serves the largest geographic region of all NZ's DHBs (https://www. southerndhb.govt.nz/index.php?page=654). Key informants were sampled purposively in order to construct a maximum variation sample that aimed to include primary care (GPs), secondary care clinicians, community nursing services and health service planners from the Southern DHB and WellSouth PHO. From this group of stakeholders, we also aimed to include those who had a Southern DHB planning/management, Alliance South or other clinical leadership role, had direct involvement with the Alliance South HealthPathways work programme or who were end users of the HealthPathways (GPs).

\section{Data collection}

The semistructured interviews used a topic guide based on a literature review, relevant CFIR guidance (https:// cfirguide.org/) and discussions within the research team. The topic guide (online supplementary file 1) covered: (A) views concerning the acceptability and utility of HealthPathways; (B) the implementation of the HealthPathways initiative in Southern Region, with a focus on context-specific barriers and facilitators to implementation; and (C) any specific issues relating to the actual use of HealthPathways by participants (if a healthcare 


\section{Box 1 CFIR domains and associated constructs.}

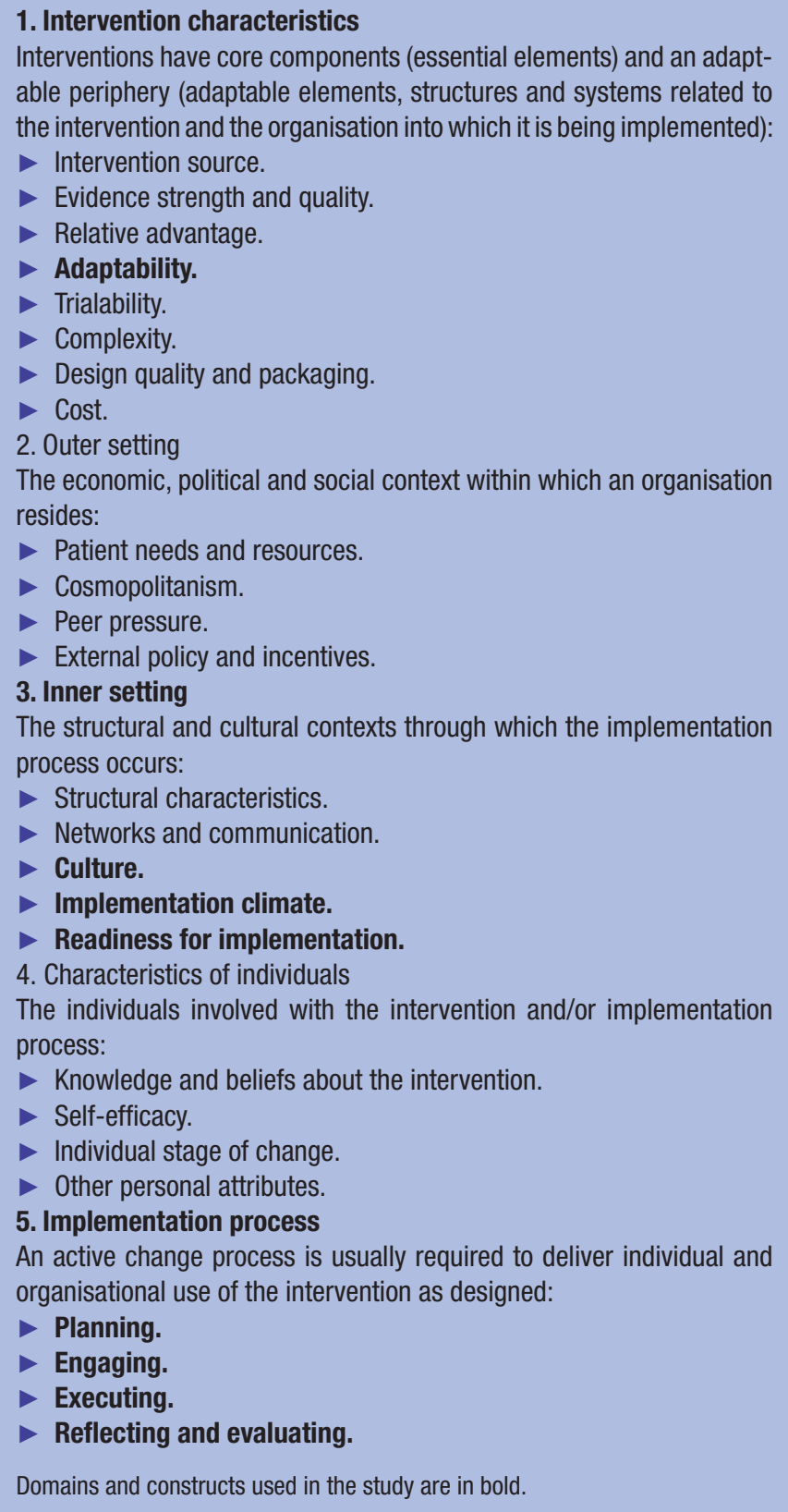

practitioner). The topic guide was used flexibly to allow participants to construct their accounts in their own terms. All interviews were digitally recorded and transcribed verbatim.

\section{Data analysis}

A deductive thematic analysis was conducted using the framework method. ${ }^{18}$ Interviews were coded deductively by ET, assisted by NVivo 10 qualitative analysis software, into the five domains of the CFIR (http://www.cfirguide. $\mathrm{org} /$ ) and, as appropriate, into each domains' constructs. TS independently checked the assignment of a sample of data to the domains and constructs. Interpretation of the data, in particular the linkage between the CFIR domains and constructs, was an iterative process that was led by
ET and TS, with input from FD-N and RG. In addition, reports and relevant documents were sought from the Alliance South Southern HealthPathways Steering Group to provide background information and timelines.

The Consolidated criteria for Reporting Qualitative research ${ }^{19}$ was used to structure reporting of the methods and the findings (online supplementary file 2 ).

\section{Patient and public involvement \\ No patients or the public were involved in this study.}

\section{RESULTS}

Five GPs and five secondary care clinicians were interviewed. Of the 10 participants, 4 had a Southern DHB planning/management, Alliance South or other clinical leadership role. Three participants had direct involvement with the Alliance South HealthPathways work programme. Three participants were end users of HealthPathways.

As the interviews progressed and analysis was undertaken, it became apparent that there were significant problems with the implementation of HealthPathways in the Southern Region. Thus, the focus of the analysis shifted to using the relevant CFIR domains and constructs (box 1) to systematically explore the question as to why implementation was proving problematic. Illustrative participant quotes are presented.

\section{Domain 1: intervention characteristics}

Core component and adaptable periphery

The CFIR (box 1) conceptualises complex interventions as having both core components (defined as the essential and indispensable elements of the intervention itself) and an adaptable periphery (adaptable elements, structures and systems related to the intervention and the organisation into which it is being implemented). ${ }^{14}$ In order for an intervention to be implemented successfully, account must be taken of both the core components and the adaptable periphery.

Participants reported that the core component of the HealthPathways intervention-the web portal and referral system that contained a large number of individual clinical care pathways-had been addressed well by the product developers. It was considered to be well designed and easy to access, the pathways for each clinical condition were appropriate and the web portal supported use of the ERMS that generated the referral letter from primary to secondary care:

This has changed my life in primary care. This is the best thing .... If I have a problem, if I have a woman come in with postmenopausal bleeding I go tick, tick, tick, there it is. 'Have you done this, this, and this? Is it this or that?' Do that. If it is this and this, then refer here [ERMS] and she will get an appointment. It's just e-mail off, she'll get an appointment within three weeks. (Participant 4) 
On the whole I'm a big fan of HealthPathways. I'm a fan of it and ERMS linking up well together .... General Practice in New Zealand is probably more electronically savvy than any other place in the world. (Participant 5)

What was apparent, however, in participants' accounts of how the core component (web portal and referral system) was implemented was there was limited or no consideration of the adaptable periphery. There was no clear understanding by the local team leading the implementation process of the need to both construct and populate a web portal and referral system (core component) and to address related issues that were necessary for the Canterbury HealthPathways to be successfully adapted and used in the Southern Health System (adaptable periphery). As a consequence, the implementation project was resourced to deliver the web portal and referral system but not to do the necessary related activities. Thus, there was little or no funding of primary care time to allow engagement with project development and with secondary care. Support for the key activity of communicating about the intervention, its benefits and how it can be used to further integration across primary and secondary care was also lacking:

I don't think you can go back to what Canterbury did [to achieve successful implementation] because the time and resources aren't there. (Participant 3)

In short, it was apparent that the adaptable periphery had not been addressed; it was seen as simply sufficient to deliver the web portal and referral system populated with a minimally edited set of individual clinical care pathways (the core component of HealthPathways) to effect successful implementation:

What they would do would be to take the Pathways that had been developed in Canterbury and simply cross out Canterbury and write Southern on them, and that would be fine and behaviours would change and everything would be hunky-dory. This mistaken view that what's on the flowchart on the piece of paper at the end is the process, which it isn't. I said to them on numerous occasions it is like taking somebody else's holiday snapshots and thinking that you've then had a holiday, which is complete nonsense. Most of them you won't understand. The essential part of HealthPathways is the conversation. (Participant 1)

\section{Domain 3: inner setting}

CFIR's 'inner setting' (box 1) is defined as the structural and cultural contexts through which the implementation process occurs. $^{14}$

\section{Culture}

Implementation of the HealthPathways took place in a local health system where two strong corporate and professional cultures worked against the successful implementation of an intervention that straddled both primary and secondary care. The first of these, the local corporate culture-represented through the relationships between the DHB, PHO, secondary care clinicians and general practice-was seen as being both resistant to change and characterised by a low trust relationship between the DHB and other actors in the system. Resistance to change was considered at least in part due to a continued environment of financial austerity and a need for the DHB to 'balance its books':

A resistance to change, which I think was about the whole health system being so stressed at the time with focus on financial deficit. (Participant 8)

A low trust relationship was considered to exist between the DHB and the other actors, notably general practice. Participants considered HealthPathways was yet another transient initiative to improve healthcare, which would likely fail to deliver its intended outcomes:

Mistrust, it's being done to us and what are the DHB, they're sending me negative vibes about management in this DHB. This is another effort and just one of their things, and like other things it will come and go. (Participant 2)

The second prevalent culture was one where secondary care (hospital specialists) and primary care (GPs) were seen as having different cultures based on their different scopes of medical practice and funding models. This culture of an embedded division between primary and secondary care had not to date been addressed in the Southern Region through formal facilitated initiatives to get both groups working collaboratively across primary and secondary care:

I don't think that there's any particular antipathy. The way I see it from the secondary care perspective, by and large, and I don't really know what our colleagues in primary care think, but I do think that from secondary care clinicians, they've never had the opportunity of being in the same room and working out processes with primary care. I don't think they're opposed to it, it's just a foreign idea. (Participant 1)

\section{Implementation climate}

A key aspect of the implementation climate that was not addressed properly was organisational incentives and rewards. The implementation process failed to consider ways in which using the HealthPathways would be rewarded for either secondary or primary care. That is to say, for clinicians that understood the purpose of pathways, then using them made sense and was rewarding because there was an appreciation that they made clinical processes more efficient. For clinicians who had not yet bought into pathways, not using them meant business as usual (ie, no direct penalty was incurred other than having to either write referrals that were denied or receive referrals that did not include all the information). Thus, there was little incentive to engage with the intervention 
that required getting used to a new system and undertaking extra effort (ie, for GPs more tests to be done prior to referral to secondary care; for hospital specialists more explicit triaging of referrals based on the specific clinical pathway criteria):

I know the secondary care champion on the steering group is finding it very difficult to engage other clinicians. Until there's some pressure to treat people, or to manage and triage people according to HealthPathways there, then you won't get the buy-in. (Participant 9)

\section{Readiness for implementation}

This CFIR construct is defined as the tangible and immediate indicator of the organisation's decision to implement an intervention ${ }^{14}$ (in this case the organisation is defined as the DHB and the PHO working together through an Alliancing framework). It consists of three subconstructs (leadership engagement, available resources and access to information and knowledge). Overall, readiness for implementation was limited. Apart from the HealthPathways steering group, which was engaged with the project, there was variable leadership engagement with primary and secondary care. In terms of access to information and knowledge about HealthPathways, this was strong in primary care, but less so in secondary care:

Buy-in is very limited from the secondary care system, whereas the PHO have helped to put HealthPathways on everyone's workstation, or nearly everyone's workstation, and so at least it's there, available to use, and there's some buy-in that way. (Participant 3)

As already explored in domain 1, lack of available resources was a main reason why there was a lack of readiness to implement HealthPathways. Most participants considered an appropriately funded adaptation would have included dedicated time for secondary and primary care to meet and interact meaningfully. This had not been the case:

The key to it [Canterbury HealthPathways] was that people in the primary care sector were able to sit down in a room with secondary care clinicians and talk about the experience for patients and what that meant. (Participant 1)

\section{Domain 5: the implementation process}

Participants considered that there had been multiple failures of the implementation process (box 1) across all its four components: planning, engaging, executing and reflecting/evaluating.

\section{Planning}

This was perceived as an omission by participants. They considered that those who set up the programme to take the Canterbury HealthPathways and adapt them for use in the Southern region had only planned for the technical production of a suite of adapted pathways and a basic 'roll out', with a limited amount of promotion. This was considered to show a lack of understanding about what was achieved in Canterbury during the planning phase and what was important to the successful uptake and use of HealthPathways. Various initiatives were then undertaken after the fact with champions appointed in secondary and primary care and communications disseminated more regularly across the health sector.

\section{Engagement}

Engagement with the actual users of the HealthPathways was limited. Participants raised the issue of a lack of planning to ensure that the wider community in primary and secondary care were given the opportunity to meaningfully engage with the HealthPathways initiative, which one participant referred to as 'socialisation':

Then I think the hardest part is probably how do you actually socialize it amongst clinicians so that they regularly use the pathways. (...) I think that's probably where things have not gone as well as they need to or could have. (Participant 7)

It's an uphill battle to get access to my secondary care colleagues, and when I meet up with them, the first question I ask is, 'How many of you know about HealthPathways?' One or two might put up their hands. 'How many of you use it?' Then it's sort of deafening silence. A huge lack of knowledge about what HealthPathways are and how they can benefit everybody, primarily the patient, but they can help all of us in what we do. A lack of knowledge, and there's been still no concerted drive from the powers that be to propagate the concept of HealthPathways and how everybody can benefit from it. (Participant 2)

Although a secondary care and a primary care clinician (hospital specialist and GP) had been identified as champions for Pathways (both formally and informally), their work was frustrated by a lack of managerial support during the implementation process:

If you have something taken from outside and imposed on you, without any background and information as to how you can benefit from this, individuals like me trying to propagate that, I can easily be seen as just somebody the DHB has put there to try and wave the flag. With no support from above, I'm fighting a losing battle. (Participant 2)

\section{Executing}

Executing the implementation was problematic, as explored in domain 1, because it focused on the core component and not on the adaptable periphery. What this meant was that significant work was devoted to technology development (website, web portal and localising existing individual care pathways), thus the Clinical Editors were contracted by the DHB to produce a large suite of edited pathways, but there was inadequate resourcing to allow 
meaningful engagement with the wider secondary care community. As a consequence, 'buy in' from clinicians was limited.

\section{Evaluation/reflection}

There appeared to be very limited evaluation of the Southern HealthPathways implementation. The only data that was reported were regular updates on the number of live HealthPathways. Information on the numbers of health practitioners actually using the individual clinical pathways was not consistently reported across specialty areas.

\section{DISCUSSION}

This is the first process evaluation of HealthPathways implementation in NZ using qualitative methods and the first such evaluation to use the CFIR. The use of implementation science theory (CFIR) allowed us to 'unpack' the reasons why the implementation of HealthPathways in the Southern Region of NZ was so challenging. CFIR postulates that for an intervention to be implemented successfully, account must be taken of both the intervention's core components and the adaptable periphery. We found that the core component of HealthPathwaysthe web portal and referral system that contains a large number of individual clinical care pathways-had been addressed well by the product developers. Little attention had, however, been paid to addressing the adaptable periphery (adaptable elements, structures and systems related to the intervention and the organisation into which it is being implemented); it was seen as sufficient just to deliver the web portal and referral system populated with a set of localised clinical care pathways as developed to effect successful implementation. In terms of CFIR's 'inner setting' corporate and professional cultures, the implementation climate and readiness for implementation were not properly addressed during implementation. There was a culture of low trust between the DHB and other actors in the health system and an embedded division between primary and secondary care clinicians. There were no organisational rewards or incentives for end users to use HealthPathways (poor implementation climate). There was also a lack of readiness to implement, notably a lack of funding to allow for dedicated time for secondary and primary care clinicians to meet and interact meaningfully as part of HealthPathway development. There were also multiple failures of the implementation process across all its four components: planning, engaging, executing and reflecting/evaluating.

This qualitative interview study used purposive sampling to recruit a sample that varied by healthcare professional group (primary care and secondary care) and by degree of involvement with the Southern HealthPathways programme. The choice of individual interviews was appropriate as it allowed participants to talk openly about the problems being encountered with the HealthPathways programme. It was appropriate that the study focused on a single NZ health region as it was designed in partnership with the local health system (Alliance South) to provide context specific findings that would be of benefit to the local health system. ${ }^{20}$ Nonetheless, it is likely that our key finding - the need to consider the adaptable periphery when implementing interventions-is transferable to other NZ health regions and other health systems in which HealthPathways are being implemented. A strength of the chosen implementation science framework (CFIR) is that it is "meta-theoretical', including constructs from a synthesis of existing implementation theories, and it is designed to allow researchers to test out empirically mechanisms theorised to promote or hinder implementation of a given intervention. ${ }^{14}$ It is also intended to be used flexibly, with the researchers selecting and identifying constructs that best explain their findings. We did not have an a priori view of which CFIR domains would explain the problems in the implementation process, we deductively coded into all of the five CFIR domains and through an iterative process of reviewing the domain categories we were able to identify those which were most relevant to HealthPathways implementation and thereby explain why the implementation had been problematic in this specific context.

We found the recruitment of participants challenging and, while we had not set a fixed number of planned interviews, were only able to recruit a small number of participants (three) who were categorised as end users of HealthPathways and not involved with the implementation process. We believe this difficulty reflects the findings, in that the project was not well planned and implemented and so interest in speaking about HealthPathways was adversely affected. We had originally intended to combine this qualitative process evaluation with a quantitative component to determine if HealthPathways were effective in improving care of people with two exemplar clinical conditions through a before and after comparison of key process and outcome measures. We were unable to meet this second objective as we were informed, after award of the research grant, that the HealthPathways team would not be able to collect these data due to delays in implementing the project and project planning. It was a requirement of the study that Alliance South would set up the data collection process, not the research team.

There has to date been no formative evaluation of HealthPathways in NZ. The Canterbury HealthPathways programme, for example, has conducted a limited evaluation consisting of an online survey of primary and secondary care clinicians and audits of pathways use. ${ }^{3921}$ In contrast, two different Australian states have conducted a process evaluation of HealthPathways using a qualitative case study approach: New South Wales (Hunter and New England) ${ }^{5}$ and Victoria (Barwon). ${ }^{611}$ Neither Australian studies used an explicit implementation science framework; instead, they descriptively list the 'barriers' and 'facilitators' to implementation. These two evaluations considered that implementation had been successful, and the Hunter and New England study's list of 'critical 
success factors' are the mirror image of a number of the key implementation issues identified in this study: senior management support, senior clinical leadership, involvement of clinicians in development, need for pathways to add value for clinicians and engagement with hospital specialists. ${ }^{5}$ Both evaluations' list of 'barriers' highlighted lack of awareness by GPs of the HealthPathways programme. ${ }^{56}$

CFIR now has a good evidence base as it has been applied across a wide variety of implementation study designs and settings. ${ }^{15}$ A recent CFIR-informed qualitative process evaluation study (exploring the barriers affecting implementation of an online frailty tool into primary care), using a similar methodology, also found that it was necessary to report empirical findings that related to the most important CFIR domains to best 'make sense' of the data. ${ }^{22}$

Given we intended that this evaluation should help Alliance South in its implementation of HealthPathways in line with a university-health sector collaboration, ${ }^{20}$ we are able to report how these findings have been used. During the study interview phase (2016), we were able to feedback highlights of our emerging findings to the Alliance South HealthPathways workstream who then shared with us their own internal report (Bridget-Mary McGown, Alliance South, personal communication). The latter report independently highlighted several of our emerging findings. On completion of our preliminary analysis (2017), our findings were presented to the Alliance South HealthPathways Steering Group. At the same time, the DHB had undergone a restructure with a new chief executive who wished to prioritise HealthPathways implementation. There was therefore senior management team support to initiate a relaunch of the programme, which has taken account of the key findings of this study, notably the need to ensure dedicated time for secondary and primary care clinicians to meet and interact as part of HealthPathways development.

It is not always the case that quality improvement initiatives, such as HealthPathways, are successfully implemented and thereby lead to positive outcomes for patients and health practitioners. Publication bias may also mean that there are less published studies reporting negative quality improvement research findings. ${ }^{23} \mathrm{We}$ argue there is great benefit in understanding why, and under what circumstances and in which contexts, some quality improvement interventions fail. Although we currently have limited evidence as to whether HealthPathways improve health outcomes for patients (as opposed to being viewed by health practitioners as improving referral quality and promoting integration of care), it is clear that there is rapid adoption of HealthPathways both within other regions of NZ, Australia and also in the UK. ${ }^{6}$ A consequence of this is that they are being implemented in very different contexts than those present in Canterbury NZ in 2007-2008 when the programme was first developed and implemented. What we have shown, using the CFIR framework, is that a focus on the core component of HealthPathways (web portal and referral system) without adequate consideration of the adaptable periphery (which is context dependent) when implementing HealthPathways into a health system means implementation will likely fail. Others have similarly shown the importance of the local context in determining whether local implementation works or fails when rolling out a national quality improvement programme in secondary care.$^{24}$ We therefore consider that the implication of our work for future implementers of HealthPathways and similar complex interventions is always to consider the local context within which they are to be implemented and to tailor the implementation approach to address these.

Acknowledgements The authors would like to thank the participants for their time and the Southern DHB HealthPathways Steering Committee for their sharing of information.

Contributors TS conceived and designed the study with input from RG and FD-N. ET conducted the interviews and led on the data analysis with input from TS. TS drafted the manuscript. All authors read, provided critical review and approved the final manuscript.

Funding University of Otago Research Grant and the Dean's Bequest Fund, Dunedin School of Medicine.

Disclaimer The funding body had no involvement in the design of the study and collection, analysis and interpretation of data and in writing the manuscript.

Competing interests None declared.

Patient consent Not required.

Ethics approval Ethical approval was obtained from the Otago University Human Ethics Committee (16/024). Written informed consent was obtained from all participants.

Provenance and peer review Not commissioned; externally peer reviewed.

Data sharing statement Full deidentified interview transcripts will not be shared. Informed consent, in line with the approving ethics committee, only allows for the use of deidentified extracts within research reporting and writing, in order to maintain the privacy of participants based in a defined regional area and population, thus making their identification with full transcripts more likely.

Open access This is an open access article distributed in accordance with the Creative Commons Attribution Non Commercial (CC BY-NC 4.0) license, which permits others to distribute, remix, adapt, build upon this work non-commercially, and license their derivative works on different terms, provided the original work is properly cited, appropriate credit is given, any changes made indicated, and the use is non-commercial. See: http://creativecommons.org/licenses/by-nc/4.0/.

\section{REFERENCES}

1. Armitage GD, Suter E, Oelke ND, et al. Health systems integration: state of the evidence. Int J Integr Care 2009;9:e82.

2. Kinsman L, Rotter T, James E, et al. What is a clinical pathway? Development of a definition to inform the debate. BMC Med 2010;8:31.

3. McGeoch G, Anderson I, Gibson J, et al. Consensus pathways: evidence into practice. N Z Med J 2015;128:86-96.

4. Robinson S, Varhol R, Bell C, et al. HealthPathways: creating a pathway for health systems reform. Australian health review: a publication of the Australian Hospital Association 2014 (Epub ahead of print 1 Dec 2014).

5. Gray JS, Swan JR, Lynch MA, et al. Hunter and New England HealthPathways: a 4-year journey of integrated care. Aust Health Rev 2018;42:66-71.

6. Gill SD, Mansfield S, McLeod M, et al. HealthPathways improving access to care. Aust Health Rev 2018. early online publication (Epub ahead of print 9 Feb 2018).

7. Reyneke A, Jaye C, Stokes T. Local clinical pathways: from 'good ideas' to 'practicality' for general practitioners. J Prim Health Care 2018;10:215. 
8. Timmins N, Ham C. The quest for integrated health and social care. A case study in Canterbury, New Zealand. London 2013.

9. McGeoch G, McGeoch P, Shand B. Is HealthPathways effective? An online survey of hospital clinicians, general practitioners and practice nurses. N Z Med J 2015;128:36-46.

10. Ministry of Health. Better, Sooner, More Convenient Health Care in the Community. Wellington: Ministry of Health, 2011.

11. Mansfield SJ, Quirk F, von Treuer K, et al. On the right path? Exploring the experiences and opinions of clinicians involved in developing and implementing HealthPathways Barwon. Aust Health Rev 2016;40:129-35.

12. Gauld R. What should governance for integrated care look like? New Zealand's alliances provide some pointers. Med J Aust 2014;201(3 Suppl):67-8.

13. Craig P, Dieppe P, Macintyre S, et al. Developing and evaluating complex interventions: The new medical research council guidance. BMJ 2008;337:a1655.

14. Damschroder LJ, Aron DC, Keith RE, et al. Fostering implementation of health services research findings into practice: a consolidated framework for advancing implementation science. Implement Sci 2009;4:50

15. Kirk MA, Kelley C, Yankey N, et al. A systematic review of the use of the consolidated framework for implementation research. Implement Sci 2016;11:72.

16. Davidoff F, Dixon-Woods $M$, Leviton $L$, et al. Demystifying theory and its use in improvement. BMJ Qual Saf 2015;24:228-38.
17. Improved Clinical Effectiveness through Behavioural Research Group (ICEBeRG). Designing theoretically-informed implementation interventions. Implement Sci 2006;1:4.

18. Gale NK, Heath G, Cameron E, et al. Using the framework method for the analysis of qualitative data in multi-disciplinary health research. BMC Med Res Methodol 2013;13.117.

19. Tong A, Sainsbury P, Craig J. Consolidated criteria for reporting qualitative research (COREQ): a 32-item checklist for interviews and focus groups. Int J Qual Health Care 2007;19:349-57.

20. Soper B, Yaqub O, Hinrichs S, et al. CLAHRCs in practice: combined knowledge transfer and exchange strategies, cultural change, and experimentation. J Health Serv Res Policy 2013;18(3 Suppl):53-64.

21. Kenealy TW, Sheridan NF, Connolly MJ. HealthPathways website: making the right thing the easy thing to do? N Z Med J 2015;128:6-9.

22. Warner G, Lawson B, Sampalli T, et al. Applying the consolidated framework for implementation research to identify barriers affecting implementation of an online frailty tool into primary health care: a qualitative study. BMC Health Serv Res 2018;18:395.

23. Kringos DS, Sunol R, Wagner $\mathrm{C}$, et al. The influence of context on the effectiveness of hospital quality improvement strategies: a review of systematic reviews. BMC Health Serv Res 2015;15:277.

24. Dixon-Woods M, Leslie M, Tarrant C, et al. Explaining Matching Michigan: an ethnographic study of a patient safety program. Implement Sci 2013;8:70. 